12

\title{
Влияние электрон-дырочной асимметрии на электронную структуру спиральных краевых состояний в квантовой яме $\mathrm{HgTe} / \mathrm{HgCdTe}$
}

\author{
() М.В. Дурнев \\ Физико-технический институт им. А.Ф. Иофрфе РАН, \\ Санкт-Петербург, Россия \\ E-mail: durnev@mail.ioffe.ru
}

Поступила в Редакцию 14 ноября 2019 г.

В окончательной редакции 14 ноября 2019 г.

Принята к публикации 19 ноября 2019 г.

\begin{abstract}
Изучено влияние электрон-дырочной асимметрии на электронную структуру спиральных краевых состояний в квантовой яме $\mathrm{HgTe} / \mathrm{HgCdTe}$ В рамках четырехзонной $\boldsymbol{k p}$-модели, учитывающей отсутствие центра пространственной инверсии, получены аналитические выражения для энергетического спектра и волновых функций краевых состояний, компонент тензора $g$-фактора и матричных элементов электро-дипольных оптических переходов между спиновыми ветвями краевых электронов. Показано, что при одновременном выполнении двух условий - электрон-дырочной асимметрии и отсутствии центра инверсии - спектр краевых электронов отклоняется от линейного, получены выражения для поправок к линейному спектру.
\end{abstract}

Ключевые слова: Топологические изоляторы, теллурид ртути, краевые состояния, магнитное поле.

DOI: 10.21883/FTT.2020.03.49012.629

\section{1. Введение}

Изучение спиральных краевых состояний, возникающих на краю двумерных топологических изоляторов, является важной областью физики двумерных кристаллических систем с нетривиальными топологическими свойствами [1-3]. К ключевым направлениям исследований можно отнести изучение локального и нелокального электронного транспорта по краевым каналам [4-7], механизмов рассеяния назад [8-14] и фотогальванического эффекта $[15,16]$. Среди различных систем, на краях которых экспериментально обнаружены одномерные спиральные каналы $[2,3,17]$, наибольшее внимание привлекают квантовые ямы $\mathrm{HgTe} / \mathrm{HgCdTe}$ В таких ямах переход между тривиальным и топологическим состоянием происходит при изменении ширины квантовой ямы.

Для поиска электронных состояний в квантовых ямах $\mathrm{HgTe} / \mathrm{HgCdTe}$ вблизи критической ширины обычно используется четырехзонная $\boldsymbol{k p}$-модель, включающая в себя расположенные близко по энергии электронные и дырочные подзоны. Изотропный вариант этой модели носит название модели Bernevig-Hughes-Zhang (BHZ) [1] и широко используется для расчета электронной структуры объемных и краевых состояний [18-25]. Однако, как показывают атомистические расчеты [26,27], отсутствие центра пространственной инверсии в решетке цинковой обманки и низкая симметрия гетероинтерфейсов квантовой ямы $\mathrm{HgTe} / \mathrm{HgCdTe}$ приводят к сильному смешиванию электронных и дырочных подзон, которое модифицирует $\boldsymbol{k p}$-модель $[19,27,28]$ и приводит к существенной перестройке объемных и краевых электронных состояний $[27,29,30]$. Отсутствие центра пространственной инверсии в квантовой яме $\mathrm{HgTe} / \mathrm{HgCdTe}$ ответственно, например, за возникновение оптических переходов между спиральными состояниями с противоположным спином в рамках сильного электродипольного механизма [31].

Как в изотропной модели $\mathrm{BHZ}$, так и в гамильтониане нецентросимметричной $\boldsymbol{k p}$-модели присутствуют диагональные слагаемые, пропорциональные квадрату волнового вектора и связанные с наличием далеких энергетических зон. Эти вклады отличаются по величине для электронных и дырочных подзон, что приводит к нарушению электрон-дырочной симметрии: при замене электрона на дырку и одновременной смене знака энергии эффективный $\boldsymbol{k} \boldsymbol{p}$-гамильтониан не переходит сам в себя.

Несмотря на то, что квадратичные по волновому вектору слагаемые сами по себе слабо влияют на групповую скорость и ширину локализации краевых состояний - эти характеристики определяются большим межзонным смешиванием, линейным по волновому вектору, - электрон-дырочная асимметрия, заложенная в диагональных слагаемых, приводит к значительной перестройке энергетического спектра и волновых функций краевых состояний. Электрон-дырочная асимметрия может приводить также к возникновению новых эффектов. Она, например, ответственна за возникновение циркулярного фотогальванического эффекта с участием спиральных состояний $[15,24,31,32]$. Влияние электрондырочной асимметрии на спектр и структуру краевых состояний изучалось ранее в рамках модели BHZ (см., например, работы $[24,25])$. Однако подобные исследования не проводились для реалистичных квантовых ям, не обладающих центром пространственной инверсии.

В работе исследовано влияние электрон-дырочной асимметрии на спиральные краевые состояния в квантовых ямах $\mathrm{HgTe} / \mathrm{HgCdTe}$ В частности, проанализирован характер дисперсии краевых электронов и получены 


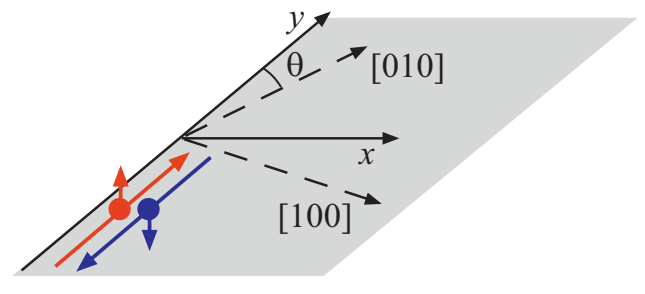

Рис. 1. Край топологического изолятора на основе квантовой ямы $\mathrm{HgTe} / \mathrm{CdHgTe} \mathrm{с} \mathrm{изображением} \mathrm{спиральных} \mathrm{краевых} \mathrm{состо-}$ яний.

выражения для волновых функций краевых состояний в рамках нецентросимметричной $\boldsymbol{k p}$-модели, найден тензор $g$-фактора краевых электронов, рассчитаны матричные элементы оптических переходов между спиновыми ветвями краевых состояний. Работа имеет следующую структуру: в разделе 2 изложена общая модель и получены выражения для энергии и волновых функций краевых состояний при нулевом волновом векторе вдоль края; в разделе 3 рассчитан тензор $g$-фактора краевых электронов; в разделе 4 найден спектр и волновые функции краевых состояний в широком диапазоне волновых векторов; в разделе 5 рассчитаны матричные элементы оптических переходов между спиновыми ветвями краевых состояний; наконец, в разделе 6 обсуждается влияние граничных условий на полученные результаты.

\section{2. Структура краевых состояний при нулевом волновом векторе}

В этом разделе мы сформулируем модель и получим волновые функции спиральных состояний при нулевом волновом векторе движения вдоль края. Рассмотрим квантовую яму $\mathrm{HgTe} / \mathrm{CdHgTe}$, выращенную вдоль кристаллографического направления [001], с шириной, близкой к критической, при которой происходит переход в фазу топологического изолятора. Такая структура обладает точечной симметрией $D_{2 d}$ без центра пространственной инверсии. Состояния вблизи уровня Ферми формируются из лежащих близко по энергии электронно-подобных подзон $|E 1, \pm 1 / 2\rangle$ и дырочноподобных подзон $|H 1, \pm 3 / 2\rangle[1]$. В базисе $|E 1,+1 / 2\rangle$, $|H 1,+3 / 2\rangle,|E 1,-1 / 2\rangle,|H 1,-3 / 2\rangle$ состояния в квантовой яме описываются следующим гамильтонианом [29]:
Здесь $\mathbf{k}=\left(k_{x}, k_{y}\right)$ - волновой вектор электрона в плоскости квантовой ямы, $k=|\mathbf{k}|, k_{ \pm}=k_{x} \pm i k_{y}, \mathcal{A}$, $\mathscr{B}, \mathscr{D}, \delta_{0}$ и $\gamma-$ вещественные параметры зонной структуры. При $\mathscr{B}<0(\mathscr{B}>0)$ и $\delta_{0}<0\left(\delta_{0}>0\right)$ квантовая яма находится в режиме топологического изолятора, и на ее краях возникают спиральные краевые состояния. Параметр $\gamma$ описывает отсутствие центра инверсии в структуре и, в основном, определяется смешиванием подзон $|E 1, \pm 1 / 2\rangle$ и $|H 1, \mp 3 / 2\rangle$ на интерфейсах квантовой ямы [27]. Значение этого параметра пока не установлено экспериментально, а теоретические значения лежат в диапазоне $2 \div 5 \mathrm{meV}$ для ямы $\mathrm{HgTe} / \mathrm{Hg}_{0.3} \mathrm{Cd}_{0.7} \mathrm{Te}[19,26-28]$.

В дальнейшем мы будем пользоваться системой координат, в которой ось $z$ совпадает с осью роста квантовой ямы, край образца параллелен оси $y$, и образец занимает полупространство $x>0$, см. рис. 1 . Ось $y$ составляет угол $\theta$ с кристаллографической осью [010], что позволяет рассматривать структуры с различными кристаллографическими ориентациями края. В частности, при $\theta=0$ край образца параллелен оси [010], в то время как при $\theta=\pi / 4$ край параллелен оси [110]. При получении аналитических выражений и анализе результатов мы будем считать, что $\mathscr{B}<0$, $\delta_{0}<0,|\mathscr{D}|<|\mathscr{B}|, \mathscr{A}>0$ и $\gamma>0$. Для численных оценок мы будем использовать набор параметров $\mathcal{A}=3.6 \mathrm{eV} \cdot \AA$, $\mathscr{B}=-68 \mathrm{eV} \cdot \AA^{2}, \mathscr{D}=-51 \mathrm{eV} \cdot \AA^{2}[19], \gamma=5 \mathrm{meV}$ [27], и $\delta_{0}=-10 \mathrm{meV}$, что соответствует яме $\mathrm{HgTe} / \mathrm{Hg}_{0.3} \mathrm{Cd}_{0.7} \mathrm{Te}$ шириной $8 \mathrm{~nm}$.

Диагональные слагаемые в гамильтониане (1), пропорциональные $k^{2}$, возникают за счет смешивания четырех рассматриваемых подзон с более энергетически далекими подзонами, которые не входят в гамильтониан. Это смешивание описывается параметрами $\mathscr{B}$ и $\mathscr{D}$. Как видно, в случае $\mathscr{D} \neq 0$ диагональные слагаемые входят несимметрично для электронных и дырочных подзон, и следовательно нарушают симметрию гамильтониана относительно замены электрона на дырку.

Таким образом, отношение $\mathscr{D} / \mathscr{B}$ можно назвать параметром электрон-дырочной асимметрии. Как будет показано дальше, спектр и волновые функции краевых состояний будут в значительной степени определяться этим параметром. Для приведенных выше параметров $\mathscr{D} / \mathscr{B} \approx 0.75$.

$$
\mathscr{H}_{0}\left(k_{x}, k_{y}\right)=\left(\begin{array}{cccc}
\delta_{0}-(\mathscr{B}+\mathscr{D}) k^{2} & i \mathscr{A} k_{+} & 0 & i \gamma e^{-2 \mathrm{i} \theta} \\
-i \mathscr{A} k_{-} & -\delta_{0}+(\mathscr{B}-\mathscr{D}) k^{2} & i \gamma e^{-2 \mathrm{i} \theta} & 0 \\
0 & -i \gamma e^{2 \mathrm{i} \theta} & \delta_{0}-(\mathscr{B}+\mathscr{D}) k^{2} & -i \mathscr{A} k_{-} \\
-i \gamma e^{2 \mathrm{i} \theta} & 0 & i \mathscr{A} k_{+} & -\delta_{0}+(\mathscr{B}-\mathscr{D}) k^{2}
\end{array}\right)
$$


Для поиска краевых состояний мы будем решать уравнение Шредингера

$$
\mathscr{H}_{0}\left(-i \frac{\partial}{\partial x}, k_{y}\right) \psi_{k_{y} s}=\varepsilon_{k_{y} s} \psi_{k_{y} s}
$$

с граничными условиями $\psi_{k_{y} s}(x=0)=0 \quad$ и $\psi_{k_{y} s}(x \rightarrow+\infty)=0$. Для каждого волнового вектора $k_{y}$ существует пара таких состояний, отличающихся значением псевдоспина $s= \pm 1 / 2$. Используемые граничные условия являются простейшими, граничные условия более сложного вида будут обсуждаться в разделе 6.

Рассмотрим вначале случай $k_{y}=0$. Покажем, что в этом случае существует аналитическое решение уравнения (2) с энергией

$$
\varepsilon_{0 s}=-\delta_{0} \frac{\mathscr{D}}{\mathscr{B}}
$$

Для этого подставим энергию (3) в уравнение (2) и запишем искомые волновые функции в виде

$$
\begin{gathered}
\psi_{0+1 / 2}=\frac{e^{i k_{y} y}}{\sqrt{2 L}}\left[\begin{array}{c}
a(x) \\
-\alpha a(x) \\
-i b(x) e^{2 i \theta} \\
-i \alpha b(x) e^{2 i \theta}
\end{array}\right], \\
\psi_{0-1 / 2}=\frac{e^{i k_{y} y}}{\sqrt{2 L}}\left[\begin{array}{c}
-i b(x) e^{-2 i \theta} \\
i \alpha b(x) e^{-2 i \theta} \\
a(x) \\
\alpha a(x),
\end{array}\right]
\end{gathered}
$$

где

$$
\alpha=\sqrt{\frac{\mathscr{B}+\mathscr{D}}{\mathscr{B}-\mathscr{D}}} .
$$

После такой подстановки уравнения на функции $a(x)$ и $b(x)$ будут аналогичны уравнениям при $\mathscr{D}=0$. Пользуясь известным решением при $\mathscr{D}=0$ [29], получим

$$
\begin{gathered}
a(x)=\mathscr{N}\left[e^{-x / l_{1}} \cos \frac{\varphi}{2}-e^{-x / l_{2}} \cos \left(k_{0} x-\frac{\varphi}{2}\right)\right], \\
b(x)=\mathscr{N}\left[e^{-x / l_{1}} \sin \frac{\varphi}{2}+e^{-x / l_{2}} \sin \left(k_{0} x-\frac{\varphi}{2}\right)\right],
\end{gathered}
$$

где

$$
\begin{gathered}
\mathscr{N}=\frac{2}{\sqrt{l_{2}\left(1+\alpha^{2}\right)}}, \\
l_{1}=-\frac{\varkappa \mathscr{B}}{\mathscr{A}}, \quad l_{2}=-\frac{\mathcal{A}}{\varkappa \delta_{0}}, \quad k_{0}=\frac{\gamma}{\mathscr{A}}, \quad \tan \varphi=-\frac{\gamma}{\varkappa \delta_{0}},
\end{gathered}
$$

и

$$
\varkappa=\frac{\sqrt{\mathscr{B}^{2}-\mathscr{D}^{2}}}{|\mathscr{B}|} .
$$

При выводе уравнения (6) использовалось соотношение $l_{1} \ll l_{2}$, которое выполнено в реалистичных квантовых ямах. Так, из (8) следует, что для приведенных выше параметров $l_{1} \approx 10 \AA, l_{2} \approx 540 \AA$. В дальнейшем, при получении результатов мы будем считать соотношение $l_{1} \ll l_{2}$ выполненным. Формулы (3)-(9) являются обобщениями результатов работы [32] (случай $\mathscr{D} \neq 0, \gamma=0$ ) и работы [29] (случай $\mathscr{D}=0, \gamma \neq 0$ ). Из полученных формул видно, что несмотря на малость параметров $\mathscr{B}$ и $\mathscr{D}$ (что проявляется в малости длины $l_{1}$ ), все результаты существенно зависят от отношения $\mathscr{D} / \mathscr{B}$.

Ключевое следствие электрон-дырочной асимметрии - это сдвиг дираковской точки $\left(\varepsilon_{0 s}\right)$ из середины щели $(\varepsilon=0)$. Из формулы (3) следует, что дираковская точка сдвигается пропорционально отношению $\mathscr{D} / \mathscr{B}$ : при $\mathscr{D}<0$ сдвиг происходит в сторону зоны проводимости, в случае $\mathscr{D}>0-$ в сторону валентной зоны. Сдвиг дираковской точки приводит к перераспределению относительных вкладов подзон $|E 1, \pm 1 / 2\rangle$ и $|H 1, \pm 3 / 2\rangle$ в волновые функции, увеличению длины затухания $l_{2}$ и изменению скорости краевых электронов. В предельных случаях $\mathscr{D}= \pm \mathscr{B}$ дираковская точка попадает на границу энергетической щели, и краевые состояния при $k_{y}=0$ гибридизуются с объемными. Как следует из (8), в этом случае $l_{2} \rightarrow \infty$.

Спроецировав часть гамильтониана (1), в которую входят слагаемые с $k_{y}$, на волновые функции (4), получим энергию краевых состояний с точностью до линейных по $k_{y}$ вкладов

$$
\varepsilon_{k_{y} s}=-\delta_{0} \frac{\mathscr{D}}{\mathscr{B}}+2 s \hbar v_{0} k_{y},
$$

где скорость краевых электронов

$$
v_{0}=\frac{\mathcal{A}}{\hbar} \frac{\left|\delta_{0}\right| \varkappa^{2}}{\sqrt{\varkappa^{2} \delta_{0}^{2}+\gamma^{2}}} .
$$

\section{3. Эффект Зеемана для краевых состояний}

В рамках рассматриваемой $\boldsymbol{k} \boldsymbol{p}$-модели взаимодействие электрона с магнитным полем В характеризуется суммой орбитального вклада, описываемого гамильтонианом (1) с подстановкой Пайерлса $\mathscr{H}_{0}[\mathbf{k}-(e / c \hbar) \mathbf{A}]$, где $\mathbf{A}-$ векторный потенциал магнитного поля, и зеемановского вклада [26]

$$
\mathscr{H}_{Z}=\frac{\mu_{B}}{2}\left(\begin{array}{cccc}
g_{e}^{\perp} B_{z} & 0 & g_{e}^{\|} B_{-} & 0 \\
0 & g_{h}^{\perp} B_{z} & 0 & g_{h}^{\|} e^{-4 i \theta} B_{+} \\
g_{e}^{\|} B_{+} & 0 & -g_{e}^{\perp} B_{z} & 0 \\
0 & g_{h}^{\|} e^{4 i \theta} B_{-} & 0 & -g_{h}^{\perp} B_{z}
\end{array}\right),
$$

где $g_{e}^{\|}, g_{e}^{\perp}, g_{h}^{\|}$и $g_{h}^{\perp}-g$-факторы подзон $|E 1\rangle$ и $|H 1\rangle$, содержащие вклады от смешивания с далекими электронными и дырочными подзонами, $B_{ \pm}=B_{x} \pm i B_{y}$, и $\mu_{B}$ - магнетон Бора. 
Взаимодействие магнитного поля с краевыми электронами в базисе $\left(\psi_{0+1 / 2}, \psi_{0-1 / 2}\right)$ описывается эффективным гамильтонианом

$$
\mathscr{H}_{\text {edge }}^{(\mathbf{B})}=\frac{\mu_{B}}{2} \sum_{\alpha, \beta=x, y, z} g_{\alpha \beta} \sigma_{\alpha} B_{\beta},
$$

где $g_{\alpha \beta}$ - компоненты тензора $g$-фактора краевых электронов, и $\sigma_{x}, \sigma_{y}, \sigma_{z}$ - матрицы Паули. Спроецировав гамильтониан (12) на волновые функции (4), получим компоненты $g$-фактора для поля В в плоскости ямы

$$
\begin{gathered}
g_{x x}=g_{1} \cos ^{2} 2 \theta+g_{2} \sin ^{2} 2 \theta, \\
g_{y y}=g_{1} \sin ^{2} 2 \theta+g_{2} \cos ^{2} 2 \theta, \\
g_{x y}=g_{y x}=\frac{1}{2}\left(g_{1}-g_{2}\right) \sin 4 \theta,
\end{gathered}
$$

где

$$
\begin{gathered}
g_{1}=\frac{1}{2}\left(\frac{\mathscr{B}-\mathscr{D}}{\mathscr{B}} g_{e}^{\|}-\frac{\mathscr{B}+\mathscr{D}}{\mathscr{B}} g_{h}^{\|}\right), \\
g_{2}=\frac{1}{2}\left(\frac{\mathscr{B}-\mathscr{D}}{\mathscr{B}} g_{e}^{\|}+\frac{\mathscr{B}+\mathscr{D}}{\mathscr{B}} g_{h}^{\|}\right) \frac{\left|\delta_{0}\right| \varkappa}{\sqrt{\varkappa^{2} \delta_{0}^{2}+\gamma^{2}}} .
\end{gathered}
$$

Формула (15) является обобщением результатов работы [29] на случай $\mathscr{D} \neq 0$. Поскольку $g$-фактор тяжелой дырки $g_{h}^{\|}$близок к нулю в квантовых ямах из материалов с решеткой цинковой обманки [33], то основной вклад в $g_{1}$ и $g_{2}$ дается первыми слагаемыми в (15). В реальных структурах множитель $(\mathscr{B}-\mathscr{D}) / \mathscr{B}$ может сильно отличаться от единицы, например, для параметров, приведенных в разделе II, $(\mathscr{B}-\mathscr{D}) / \mathscr{B} \approx 1 / 4$, то есть $g$-факторы для поля в плоскости ямы уменьшаются примерно в четыре раза по сравнению со случаем $\mathscr{D}=0$. Оценки дают $g_{1} \approx 2.5, g_{2} \approx 2$, что согласуется с результатами численного моделирования спектра краевых состояний в работе [31].

Магнитное поле, направленное по нормали к квантовой яме, смешивает подзоны $|E 1\rangle$ и $|H 1\rangle$, что приводит к большим орбитальным вкладам в компоненты $g_{\alpha z}$. Диагональная компонента $g_{z z}$, как и в случае $\mathscr{D}=0$, зависит от калибровки векторного потенциала и может быть выбрана нулем [29], в то время как недиагональные компоненты не зависят от калибровки и имеют следующий вид:

$$
g_{x z}=-g_{3} \sin 2 \theta, \quad g_{y z}=g_{3} \cos 2 \theta
$$

где

$$
g_{3}=\frac{2 m_{0} \mathscr{A}^{2}}{\hbar^{2}} \frac{\gamma\left|\delta_{0}\right| \varkappa^{2}}{\left(\delta_{0}^{2} \varkappa^{2}+\gamma^{2}\right)^{3 / 2}} .
$$

Оценки дают $g_{3} \approx 130$, то есть $g_{3} \gg g_{1}, g_{2}$.

Магнитное поле смешивает краевые состояния и открывает щель в их спектре. Величина щели при $\theta=0$

$$
\varepsilon_{g}=\mu_{B} \sqrt{g_{1}^{2} B_{x}^{2}+\left(g_{2} B_{y}+g_{3} B_{z}\right)^{2}} .
$$

Как следует из (18), щель открывается для любого направления поля за исключением случая, когда поле лежит в плоскости $y z$ и направлено под углом $-\arctan g_{2} / g_{3}$ к оси $y$. Отметим, что для любой ориентации края (любого $\theta$ ) существует направление в пространстве, вдоль которого приложенное поле не открывает щель.

Гигантская анизотропия $g$-фактора подтверждается в экспериментах по магнитотранспорту. В работе [7] обнаружено, что перпендикулярное магнитное поле приводит к подавлению проводимости по краевым каналам за счет открытия зеемановской щели, тогда как влияние продольного магнитного поля на проводимость значительно слабее.

\section{4. Структура краевых состояний при ненулевом волновом векторе}

Для того чтобы изучать электронный транспорт и оптические переходы с участием краевых электронов, необходимо знать волновые функции и спектр краевых состояний при ненулевом волновом векторе движения вдоль края. В этом разделе мы найдем спектр и волновые функции краевых состояний при $k_{y} \neq 0$. В рамках изотропной модели, соответствующей $\gamma=0$ в гамильтониане (1), а также в рамках модели без центра инверсии, но при наличии электрон-дырочной симметрии $(\gamma \neq 0, \mathscr{D}=0)$, приведены аналитические выражения для спектра и волновых функций во всем диапазоне волновых векторов. В более общем случае получены приближенные результаты, справедливые при малой величине отношения $\mathscr{D} / \mathscr{B}$ или $\gamma /\left|\delta_{0}\right|$.

\section{А. Изотропная модель}

Рассмотрим для начала случай $\gamma=0$, соответствующий изотропной модели. Детальный анализ структуры краевых состояний в изотропной модели приведен в работе [25]. В этом случае гамильтониан (1) распадается на два независимых блока размерностью $2 \times 2$. При $\gamma=0$ уравнение Шредингера (2) имеет аналитическое решение, справедливое во всем диапазоне $k_{y}[20,25]$

$$
\varepsilon_{k_{y} s}^{(0)}=-q \delta_{0}+2 s \varkappa \mathcal{A} k_{y},
$$

$$
\begin{gathered}
\psi_{k_{y}+1 / 2}^{(0)}=\frac{e^{i k_{y} y}}{\sqrt{2 L}}\left[\begin{array}{c}
a\left(x, k_{y}\right) \\
-\alpha a\left(x, k_{y}\right) \\
0 \\
0
\end{array}\right], \\
\psi_{k_{y}-1 / 2}^{(0)}=\frac{e^{i k_{y} y}}{\sqrt{2 L}}\left[\begin{array}{c}
0 \\
0 \\
a\left(x,-k_{y}\right) \\
\alpha a\left(x,-k_{y}\right)
\end{array}\right] .
\end{gathered}
$$


Здесь $q=\mathscr{D} / \mathscr{B}$, функция $a$ имеет вид [см. (6) при $\gamma=0$ ]

$$
a\left(x, k_{y}\right)=\frac{2}{\sqrt{l_{2}\left(k_{y}\right)\left(1+\alpha^{2}\right)}}\left[e^{-x / l_{1}}-e^{-x / l_{2}\left(k_{y}\right)}\right],
$$

с учетом зависимости длины локализации краевых состояний $l_{2}$ от волнового вектора

$$
l_{2}\left(k_{y}\right)^{-1}=l_{2}^{-1}-q k_{y}-k_{y}^{2} l_{1},
$$

и длины $l_{1}$ и $l_{2}$ даются формулами (8). Полученное выражение для $a\left(x, k_{y}\right)$ справедливо, как и ранее, при $l_{1} \ll l_{2}\left(k_{y}\right)$. Член $k_{y}^{2} l_{1}$ мал во всем диапазоне исследуемых $k_{y}$, поэтому в дальнейшем он не будет учитываться.

Важным следствием электрон-дырочной асимметрии $(q \neq 0)$ является зависимость длины затухания краевого состояния от $k_{y}$. Как будет показано ниже, эта зависимость приводит к ненулевому матричному элементу оператора электрического дипольного момента между краевыми состояниями.

Рассмотрим подробнее случай $q>0$. Как следует из $(22)$, длина $l_{2}\left(k_{y}\right)$ обращается в бесконечность при $k_{y}=k^{*}=1 /\left(q l_{2}\right)$ для краевого состояния с $s=+1 / 2$ и при $k_{y}=-k^{*}$ для состояния с $s=-1 / 2$. Можно показать, что в точках $k_{y}= \pm k^{*}$ дисперсионные ветви краевых состояний (19) касаются нижней границы зоны проводимости $\varepsilon_{\mathrm{c}}=\sqrt{\delta_{0}^{2}+\mathscr{A}^{2} k_{y}^{2}}$. В этих точках краевые состояния „сливаются“ с объемными (двумерными) состояниями. Однако отметим, что процессы упругого рассеяния из краевых состояний в состояния зоны проводимости включаются значительно раньше, уже при $\varepsilon_{k_{y} s}=-\delta_{0}$, то есть при $k_{y}=1 / l_{2}(1+q)$.

При движении по дисперсионным ветвям краевых состояний в сторону валентной зоны длина $l_{2}\left(k_{y}\right)$ уменьшается. При $\varepsilon_{k_{y} s}=\delta_{0}$, когда энергия краевых состояний сравнивается с энергией потолка валентной зоны, $l_{2}\left(k_{y}\right)=l_{2}(1-q)$. При дальнейшем увеличении волнового вектора $l_{2}\left(k_{y}\right)$ может стать сравнимой с $l_{1}$, и формулы (21), (22) перестают быть применимы. Аккуратный анализ этой ситуации изложен в работе [25].

\section{В. Модель без центра инверсии}

Перейдем теперь к модели реалистичных квантовых ям без центра пространственной инверсии, которая описывается гамильтонианом (1) с $\gamma \neq 0$. Мы получим аналитические результаты в двух пределах: в пределе $\gamma /\left|\delta_{0}\right| \ll 1$, когда антидиагональные члены гамильтониана (1) можно считать малым возмущением, и в пределе $|\mathscr{D}| \mathscr{B} \mid \ll 1$, то есть в пределе малой электрон-дырочной асимметрии.

\section{1. Предел $\gamma /\left|\delta_{0}\right| \ll 1$}

В этом разделе будем считать антидиагональные члены гамильтониана (1) малым возмущением.

Невозмущенные волновые функции $\psi_{k_{y} \pm 1 / 2}^{(0)}$ краевых состояний найдены в разделе 4 для произвольного $k_{y}$, см. (20). Обратим внимание, что в первом порядке теории возмущений по $\gamma$ краевые состояния $\psi_{k_{y} \pm 1 / 2}^{(0)}$ не смешиваются и не сдвигаются по энергии. Это означает, что поправки к волновым функциям краевых состояний связаны с подмешиванием к краевым состояниям объемных состояний зоны проводимости и валентной зоны.

Таким образом, в первом порядке теории возмущений по $\gamma$ энергия краевых состояний не меняется и совпадает c (19). Волновые функции краевых состояний будем искать в виде

$$
\begin{gathered}
\psi_{k_{y}+1 / 2}=\frac{e^{i k_{y} y}}{\sqrt{2 L}}\left[\begin{array}{c}
a_{1}\left(x, k_{y}\right) \\
-\alpha a_{2}\left(x, k_{y}\right) \\
-i b_{1}\left(x, k_{y}\right) e^{2 i \theta} \\
-i \alpha b_{2}\left(x, k_{y}\right) e^{2 i \theta}
\end{array}\right], \\
\psi_{k_{y}-1 / 2}=\frac{e^{i k_{y} y}}{\sqrt{2 L}}\left[\begin{array}{c}
-i b_{1}\left(x,-k_{y}\right) e^{-2 i \theta} \\
i \alpha b_{2}\left(x,-k_{y}\right) e^{-2 i \theta} \\
a_{1}\left(x,-k_{y}\right) \\
\alpha a_{2}\left(x,-k_{y}\right)
\end{array}\right],
\end{gathered}
$$

где $b_{1}$ и $b_{2}-$ искомые функции $\propto \gamma$. В первом порядке теории возмущений функции $a_{1}$ и $a_{2}$ равны и совпадают с невозмущенной функцией (21). Функция $\psi_{k_{y}-1 / 2}$ связана с $\psi_{k_{y}+1 / 2}$ операцией инверсией времени. Уравнение (2) с невозмущенной энергией (19) приводит к следующей системе уравнений на $b_{1}$ и $b_{2}$

$$
\begin{gathered}
l_{1} b_{1}^{\prime \prime}+b_{2}^{\prime}+\left[l_{2}\left(k_{y}\right)^{-1}+k_{y}\right] b_{1}+k_{y} b_{2}=-k_{0} a, \\
l_{1} b_{2}^{\prime \prime}+b_{1}^{\prime}+\left[l_{2}\left(k_{y}\right)^{-1}-k_{y}\right] b_{2}-k_{y} b_{1}=-k_{0} a .
\end{gathered}
$$

Решение этой системы, удовлетворяющее граничным условиям, имеет вид

$$
b_{1}=b(x)+B(x), \quad b_{2}=b(x)-B(x),
$$

где

$$
\begin{gathered}
b(x)=\frac{\mathscr{N}\left(k_{y}\right) k_{0} l_{2}\left(k_{y}\right)}{2}\left\{e^{-x / l_{1}}+\left[\frac{2 x}{l_{2}\left(k_{y}\right)}-1\right] e^{-x / l_{2}\left(k_{y}\right)}\right\}, \\
B(x)=-\mathscr{N}\left(k_{y}\right) k_{0} k_{y} l_{2}\left(k_{y}\right) x e^{-x / l_{2}\left(k_{y}\right)},
\end{gathered}
$$

и

$$
\mathscr{N}\left(k_{y}\right)=\frac{2}{\sqrt{l_{2}\left(k_{y}\right)\left(1+\alpha^{2}\right)}} .
$$

При $k_{y}=0$ функции $b_{1}$ и $b_{2}$ равны и совпадают с выражением (6), взятым в пределе $\gamma /\left|\delta_{0}\right| \ll 1$. Для применимости теории возмущений нужно, чтобы функции $b_{1,2}$ были малы по сравнению с $a$, то есть $k_{0} l_{2}\left(k_{y}\right) \ll 1$. Это накладывает ограничение на диапазон применимости полученных формул по $k_{y}$.

Используя полученные в первом порядке теории возмущений по $\gamma /\left|\delta_{0}\right|$ поправки к волновым функциям, 

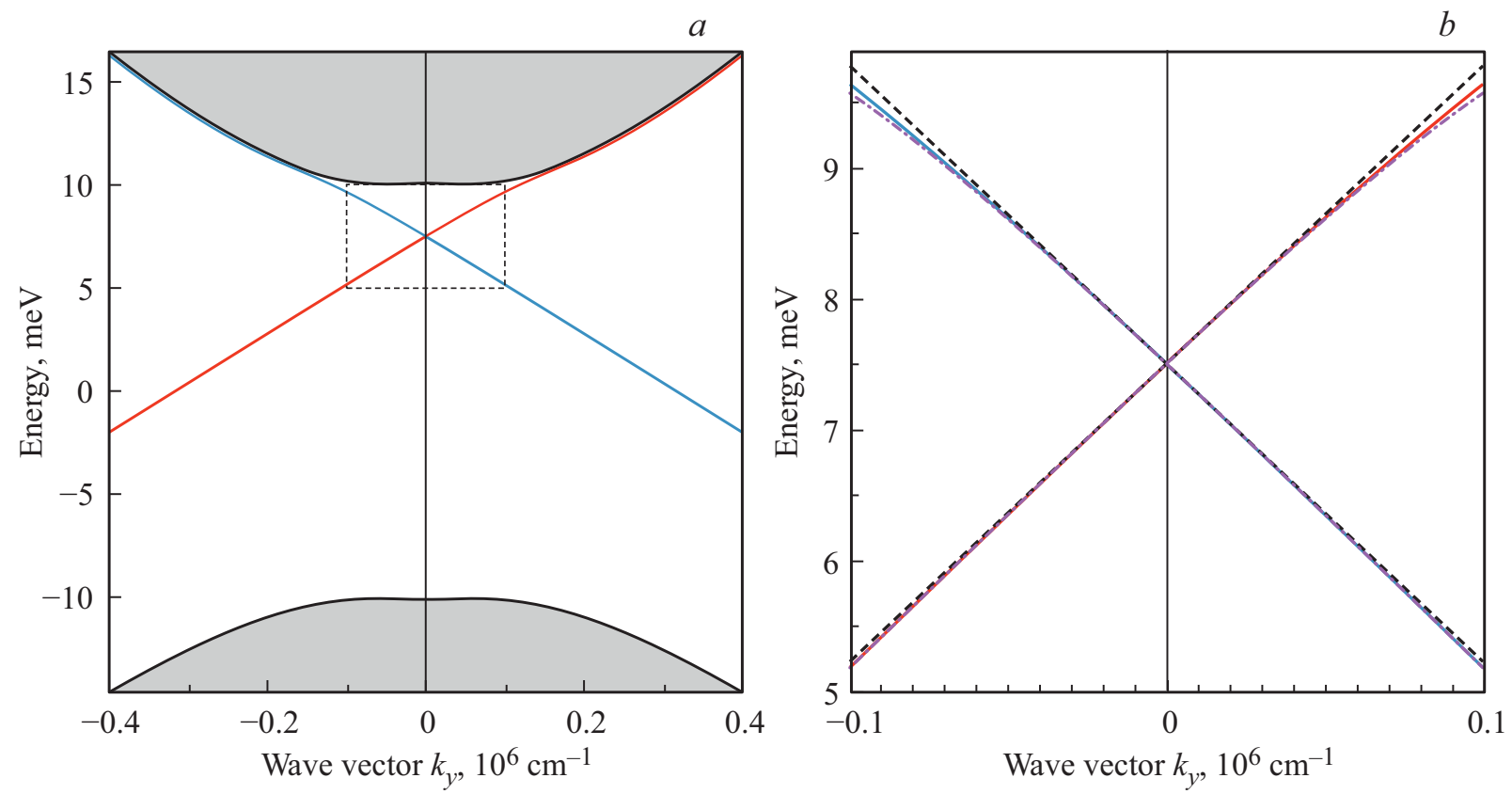

Рис. 2. Дисперсия электронных состояний в квантовой яме $\mathrm{HgTe} / \mathrm{HgCdTe}$ для зонных параметров, приведенных в тексте, и $\gamma=2 \mathrm{meV}$, так что $\gamma /\left|\delta_{0}\right|=0.2$. Ветви краевых состояний выделены. На панели $b$ ) показан увеличенный фрагмент дисперсии краевых состояний. Сплошные линии - численный расчет, штриховые линии - линейная зависимость со скоростью, рассчитанной по формуле (11), штрих-пунктирные линии - зависимость $\varepsilon_{k_{y} s}^{(0)}+\varepsilon_{k_{y} s}^{(2)}$, учитывающая отклонение от линейного поведения.

найдем поправки второго порядка к энергии краевых состояний:

$$
\varepsilon_{k_{y} s}^{(2)}=\left\langle\psi_{k_{y} s}^{(0)}\left|V_{\gamma}\right| \psi_{k_{y} s}^{(1)}\right\rangle
$$

где $\psi_{k_{y} s}^{(0)}$ - волновые функции нулевого приближения $(20), \psi_{k_{y} s}^{(1)}$ - линейная по $\gamma$ часть волновых функций (23), и $V_{\gamma}$ - антидиагональная часть гамильтониана (1). Расчет показывает, что

$$
\varepsilon_{k_{y} s}^{(2)}=-\frac{\gamma^{2}}{2\left|\delta_{0}\right|} \frac{k_{y} l_{2}}{\left(1-q k_{y} l_{2}\right)^{2}} .
$$

Формула (28) содержит как квадратичную по $\gamma$ поправку к скорости краевых электронов, так и, в случае $q \neq 0$, члены более высоких степеней по $k_{y}$. Таким образом, при одновременном выполнении двух условий - электрон-дырочной асимметрии и отсутствии центра инверсии $(q \neq 0$ и $\gamma \neq 0)$ - спектр краевых электронов отклоняется от линейного. Как и прежде, формула (28) применима в случае $k_{0} l_{2}\left(k_{y}\right) \ll 1$, то есть при $1-q k_{y} l_{2} \gg k_{0} l_{2}$.

На рис. 2 приведены результаты численного расчета краевых и объемных состояний в яме $\mathrm{HgTe} / \mathrm{CdHgTe}$ и сравнение с полученными аналитическими зависимостями. Видно, что спектр краевых электронов отклоняется от линейного со скоростью (11), и это отклонение хорошо описывается зависимостью $\varepsilon_{k_{y} s}=\varepsilon_{k_{y} s}^{(0)}+\varepsilon_{k_{y} s}^{(2)}$. Поправки к линейной дисперсии краевых электронов исследовались также в работе $[25]$ в рамках изотропной модели, но с граничными условиями более сложного вида. Нелинейность спектра приводит, например, к генерации краевого фототока в краевых каналах за счет непрямых оптических переходов [16].

\section{2. Предел малой электрон-дырочной асимметрии}

В случае произвольной величины $\gamma$, но при $q=\mathscr{D} / \mathscr{B} \ll 1$ можно также получить аналитические выражения для краевых состояний. По-прежнему, будем искать волновые функции краевых состояний в виде (23). Рассмотрим подробно состояние $\psi_{k_{y}+1 / 2}$. Волновая функция состояния $\psi_{k_{y}-1 / 2}$ связана с $\psi_{k_{y}+1 / 2}$ формулой (23), а энергия $\varepsilon_{k_{y}-1 / 2}=\varepsilon_{-k_{y}+1 / 2}$. Уравнение Шредингера (2) на функцию $\psi_{k_{y}+1 / 2}$ приводит к следующей системе

$$
\begin{gathered}
l_{1} a_{1}^{\prime \prime}+a_{2}^{\prime}+\left(l_{2}^{-1}+\frac{E}{\alpha \mathcal{A}}\right) a_{1}-k_{y} a_{2}-k_{0} b_{2}=0, \\
l_{1} a_{2}^{\prime \prime}+a_{1}^{\prime}+\left(l_{2}^{-1}-\frac{\alpha E}{\mathscr{A}}\right) a_{2}+k_{y} a_{1}-k_{0} b_{1}=0, \\
l_{1} b_{1}^{\prime \prime}+b_{2}^{\prime}+\left(l_{2}^{-1}+\frac{E}{\alpha \mathcal{A}}\right) b_{1}+k_{y} b_{2}+k_{0} a_{2}=0, \\
l_{1} b_{2}^{\prime \prime}+b_{1}^{\prime}+\left(l_{2}^{-1}-\frac{\alpha E}{\mathcal{A}}\right) b_{2}-k_{y} b_{1}+k_{0} a_{1}=0,
\end{gathered}
$$

где мы представили энергию краевого состояния в виде $\varepsilon_{k_{y}+1 / 2}=-q \delta_{0}+E$. 
Система (29) имеет аналитическое решение при $q=0$ $(\alpha=1)$ с энергией

$$
E^{(0)}=\frac{\mathscr{A} k_{y}\left|\delta_{0}\right|}{\sqrt{\delta_{0}^{2}+\gamma^{2}}} .
$$

Таким образом, спектр краевых состояний, полученный в разделе 2 по теории возмущений при малых $k_{y}$, остается в случае $q=0$ линейным во всем диапазоне волновых векторов. Подробности решения, а также выражения для функций $a_{1,2}$ и $b_{1,2}$ приведены в приложении.

При $q \ll 1$ с точностью до линейных по $q$ слагаемых имеем $\alpha \approx 1+q$ и $\alpha^{-1} \approx 1-q$. Таким образом, система (29) имеет такой же вид, что и при $q=0$, только теперь длина $l_{2}$ зависит от энергии

$$
l_{2}^{-1}(E)=l_{2}^{-1}-\frac{q E}{\mathscr{A}} .
$$

В этой формуле с точностью до членов, линейных по $q$, можно положить $E=E^{(0)}$, и тогда получим

$$
l_{2}^{-1}\left(k_{y}\right) \approx l_{2}^{-1}-\frac{q k_{y}\left|\delta_{0}\right|}{\sqrt{\delta_{0}^{2}+\gamma^{2}}} .
$$

Формула (32) описывает зависимость длины затухания краевого состояния $s=+1 / 2$ от $k_{y}$ при $q \ll 1$.

Чтобы найти поправки к дисперсии краевых состояний (30) при $q \ll 1$ подставим выражение (32) для $l_{2}\left(k_{y}\right)$ в правую часть формулы (30) для энергии. Получим в первом порядке по $q$

$$
E \approx E^{(0)}-q \mathcal{A}^{2} k_{y}^{2} \frac{\gamma^{2}\left|\delta_{0}\right|}{\left(\delta_{0}^{2}+\gamma^{2}\right)^{2}} .
$$

Из формулы (33) следует, что при наличии электрондырочной асимметрии спектр краевых состояний перестает быть строго линейным, и в нем появляются квадратичные по волновому вектору поправки. Этот результат согласуется с полученным в пределе $\gamma /\left|\delta_{0}\right| \ll 1$, см. формулу (28).

\section{5. Матричные элементы оптических переходов}

Возбуждение края топологического изолятора электромагнитной волной вызывает оптические переходы между спиновыми ветвями краевых состояний. Благодаря отсутствию центра пространственной инверсии в квантовых ямах $\mathrm{HgTe} / \mathrm{HgCdTe}$ оптические переходы возникают за счет электродипольного механизма [31]. Матричный элемент электрон-фотонного взаимодействия пропорционален матричным элементам оператора скорости $\mathbf{v}=\partial \mathscr{H}_{0} / \hbar \partial \mathbf{k}$ между состояниями $\psi_{k_{y} s}$ и $\psi_{k_{y}-s}[31]:$

$$
\begin{gathered}
v_{s-s}^{(x)}=\left\langle\psi_{k_{y} s}\left|v_{x}\right| \psi_{k_{y}-s}\right\rangle=u_{1} e^{-4 i s} \theta, \\
v_{s-s}^{(y)}=\left\langle\psi_{k_{y} s}\left|v_{y}\right| \psi_{k_{y}-s}\right\rangle=2 i s u_{2} e^{-4 i s} \theta .
\end{gathered}
$$

В этом разделе мы рассчитаем величины $u_{1}$ и $u_{2}$ в пределе $\gamma /\left|\delta_{0}\right| \ll 1$. Функции $\psi_{k_{y} s}$ для этого случая найдены в разделе 4, см. формулы (23), (25), (26). Расчет дает

$$
\begin{gathered}
u_{1}=\frac{v_{0} k_{0} k_{y} l_{2}^{2}\left[l_{2}\left(k_{y}\right)-l_{2}\left(-k_{y}\right)\right]}{2 \sqrt{l_{2}\left(k_{y}\right) l_{2}\left(-k_{y}\right)}}, \\
u_{2}=\frac{v_{0} k_{0} l_{2}^{2}}{\sqrt{l_{2}\left(k_{y}\right) l_{2}\left(-k_{y}\right)}}\left[1-\frac{l_{2}\left(k_{y}\right)+l_{2}\left(-k_{y}\right)}{2 l_{2}}\right],
\end{gathered}
$$

где $v_{0}=\varkappa A / \hbar-$ скорость краевых электронов при $\gamma /\left|\delta_{0}\right| \ll 1$. После упрощений получим

$$
u_{1}=v_{0} \frac{q k_{0} k_{y}^{2} l_{2}^{3}}{\sqrt{1-q^{2} k_{y}^{2} l_{2}^{2}}}, \quad u_{2}=-q u_{1} .
$$

Формулы (35), (36) справедливы при произвольной величине $q$ и для волновых векторов $k_{y}$, при которых выполняется соотношение $1-q k_{y} l_{2} \gg k_{0} l_{2}$. Также везде в расчетах, как в волновых функциях, так и в операторе скорости, положено $l_{1}=0$.

Как следует из (35), матричные элементы $u_{1}$ и $u_{2}$ отличны от нуля в меру зависимости длины затухания краевых состояний от $k_{y}$, а следовательно, обращаются в ноль если система обладает электрон-дырочной симметрией $(q=\mathscr{D} / \mathscr{B}=0)$. Можно показать, однако, что с учетом малых вкладов $\propto l_{1} / l_{2}$ матричный элемент $u_{2} \neq 0$ даже при $q=0$.

На рис. 3 приведены результаты расчетов матричных элементов $u_{1}$ и $u_{2}$ двумя методами - с помощью численной диагонализации гамильтониана (1) и по формулам (36). Из графиков видно, что в случае $\gamma /\left|\delta_{0}\right|=0.1$ аналитические расчеты хорошо согласуются с численными, однако при $\gamma /\left|\delta_{0}\right|=0.2$ уже наблюдается значительное расхождение, причем формулы (36) переоценивают величины $u_{1}$ и $u_{2}$. Одна из причин это то, что с ростом $\gamma$ уменьшается скорость краевых электронов $v_{0}$, которая входит в выражения для $u_{1}$ и $u_{2}$.

Оптические переходы между краевыми состояниями можно также охарактеризовать матричными элементами оператора дипольного момента, которые при малых $k_{y}$ имеют вид [31]:

$$
d_{s-s}^{(x)}=-2 s i e^{-4 i s \theta} D_{1} k_{y}, \quad d_{s-s}^{(y)}=e^{-4 i s \theta} D_{2} k_{y},
$$

где $D_{1,2}=e u_{1,2} /\left|k_{y} \omega_{s-s}\right|$ и $\hbar \omega_{s-s}=\varepsilon_{k_{y} s}-\varepsilon_{k_{y}-s}$. С учетом (36)

$$
\begin{aligned}
& D_{1}=\frac{e}{2} q k_{0} l_{2}^{3}=\frac{e \mathscr{D} \mathscr{B}^{2}}{2\left(\mathscr{B}^{2}-\mathscr{D}^{2}\right)^{3 / 2}} \frac{\gamma \mathscr{A}^{2}}{\delta_{0}^{3}}, \\
& D_{2}=-q D_{1}=-\frac{e \mathscr{D}^{2} \mathscr{B}}{2\left(\mathscr{B}^{2}-\mathscr{D}^{2}\right)^{3 / 2}} \frac{\gamma \mathscr{A}^{2}}{\delta_{0}^{3}} .
\end{aligned}
$$

Оценка по формуле (38) при $\gamma /\left|\delta_{0}\right|=0.1$ дает $D_{1} / e \approx 1.7 \cdot 10^{-12} \mathrm{~cm}^{2}$ и $D_{2} / e \approx-1.2 \cdot 10^{-12} \mathrm{~cm}^{2}$. Эта оценка согласуется по порядку величины с полученными в работе [31] численными оценками в случае $\gamma /\left|\delta_{0}\right|=0.5$. 

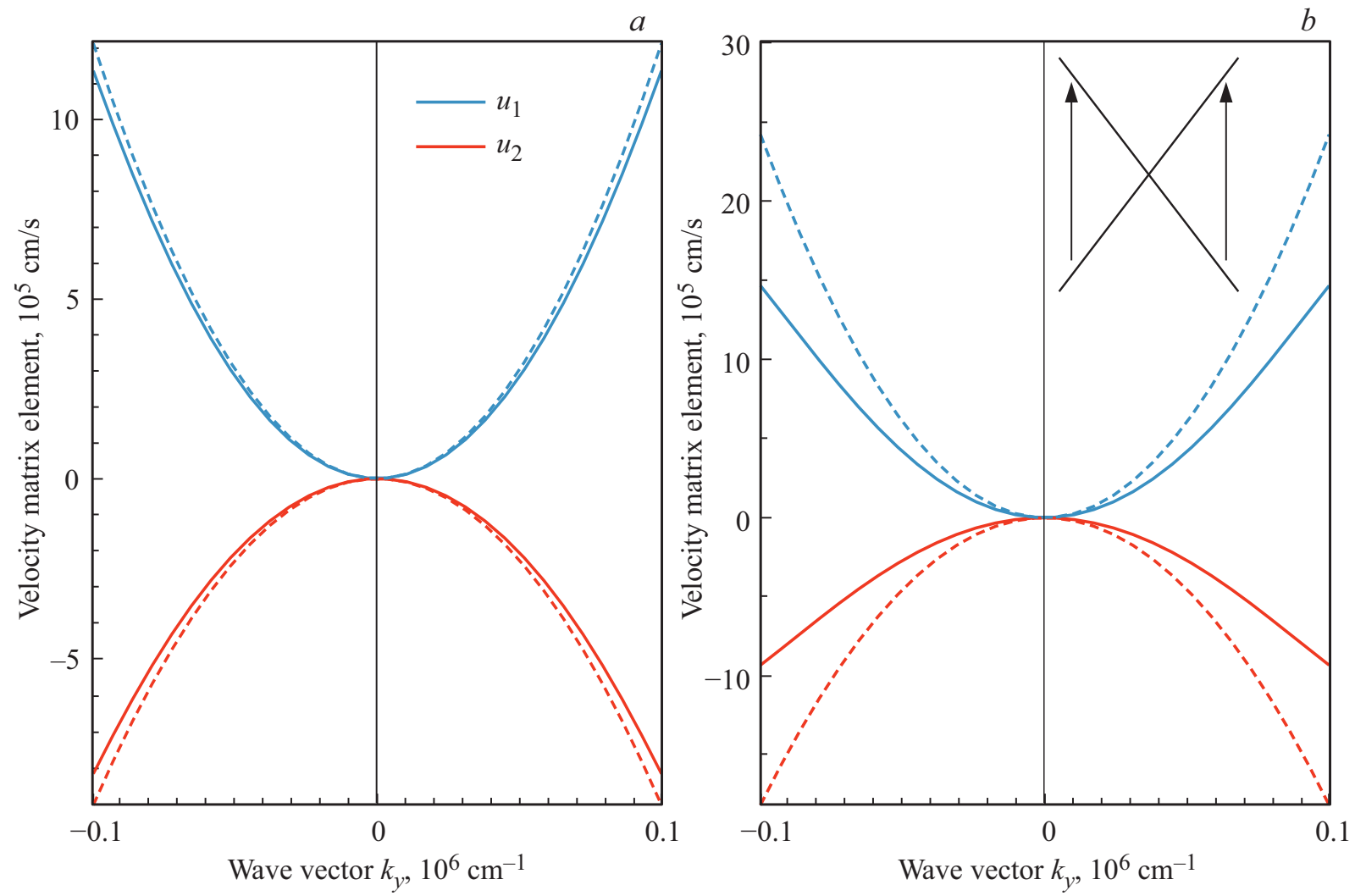

Рис. 3. Матричные элементы оператора скорости $u_{1}$ и $u_{2}$ для $\gamma /\left|\delta_{0}\right|=0.1$ (a) и $\gamma /\left|\delta_{0}\right|=0.2$ (b). Сплошные кривые - численный расчет, пунктирные - расчет по формулам (36). На вставке приведена схема оптических переходов.

При поглощении спиральными каналами циркулярно поляризованного света оптические переходы идут асимметрично в $k$-пространстве, что приводит к генерации спиновой поляризации и краевого фототока [31]. Относительная разность темпов оптических переходов из состояний $\psi_{k_{y}-1 / 2}$ и $\psi_{-k_{y}+1 / 2}, g_{k_{y}-1 / 2}$ и $g_{-k_{y}+1 / 2}$, соответственно, пропорциональна степени циркулярной поляризации падающего света $P_{\text {circ }}$ и равна

$$
\frac{g_{k_{y}-1 / 2}-g_{-k_{y}+1 / 2}}{g_{k_{y}-1 / 2}+g_{-k_{y}+1 / 2}}=K P_{\text {circ }},
$$

где

$$
K=-\frac{2 D_{1} D_{2}}{D_{1}^{2}+D_{2}^{2}}=\frac{2 \mathscr{D} \mathscr{B}}{\mathscr{B}^{2}+\mathscr{D}^{2}}
$$

Отметим, что коэффициент асимметрии $K$ для переходов между краевыми состояниями оказывается равным аналогичному коэффициенту для переходов из краевых состояний в объемные [24]. В обоих случаях $K=0$, если система обладает электрон-дырочной симметрией $(\mathscr{D}=0)$. Этот результат - следствие более общего утверждения об отсутствии спиновой поляризации и фотогальванического эффекта при поглощении циркулярно поляризованного излучения системой, обладающей электрон-дырочной симметрией [31].

\section{6. Роль граничных условий}

Результаты работы получены для простейшего „открытого“ граничного условия $\psi(x=0)=0$. Решения, полученные с более сложным граничным условием $\psi^{\prime}(x=0)+h \psi(x=0)=0$, будут отличаться от рассмотренных в работе только коэффициентами перед экспонентами $e^{-x / l_{1}}$ и $e^{-x / l_{2}}$ в функциях $a(x)$ и $b(x)$, см. например (6). В итоге, изменение параметра $h$ приводит к изменению волновых функций непосредственно вблизи края на масштабе $l_{1}$, и следовательно все полученные результаты не зависят от величины $h$, поскольку они определяются поведением волновых функциях на значительно большем масштабе $l_{2}$.

Наиболее общий вид граничных условий на границе топологического изолятора с вакуумом получен из общих физических соображений в работе [23]. Граничные условия общего вида учитывают подмешивание краем структуры далеких подзон к рассматриваемым в $\boldsymbol{k}$-модели.

В частности, возможен случай, когда граничное условие само по себе нарушает электрон-дырочную симметрию в системе. В этом случае даже при $\mathscr{D}=\mathscr{B}=0$ в гамильтониане квантовой ямы спектр краевых состояний становится асимметричным относительно центра запрещенной зоны и отклоняется от линейного $[23,35]$. 
Еще одним примером системы со спиральными состояниями является граница двух квантовых ям $\mathrm{HgTe} / \mathrm{HgCdTe}$ разной ширины, находящихся в фазе тривиального и топологического изолятора. В простейшем случае такой контакт можно смоделировать знакопеременной зависимостью $\delta_{0}(x)$ в гамильтониане $(1)$, считая, что остальные зонные параметры слабо зависят от ширины ямы. В случае $\mathscr{B}=\mathscr{D}=0$ такая модель предсказывает наличие краевых состояний с симметричным линейным спектром $[34,35]$. Однако, если $\mathscr{B} \neq 0$ и $\mathscr{D} \neq 0$, ситуация становится более сложной. Положение дираковской точки и скорость краевых электронов зависит от соотношения величин $\delta_{0}$ справа и слева от контакта. Пусть $\delta_{0}(x>0)=-\delta_{r}, \delta_{0}(x<0)=\delta_{l}$, и $\delta_{l, r}>0$. Тогда, если выполняется соотношение $\mathscr{A} / \delta_{r}, \mathcal{A} / \delta_{l} \gg l_{1}$, то электрон-дырочная асимметрия не приводит к заметному изменению спектра краевых состояний. В этом случае диагональными слагаемыми $\propto k^{2}$ в гамильтониане можно пренебречь без изменения физических результатов. Такая же ситуация реализуется в случае плавного контакта. Если же реализуется случай $\mathcal{A} / \delta_{r} \gg l_{1}$, $\mathcal{A} / \delta_{l} \lesssim l_{1}$, то электрон-дырочная асимметрия начинает играть заметную роль в спектре краевых состояний. Предел $\mathcal{A} / \delta_{l} \ll l_{1}$ соответствует ситуации, когда волновая функция не проникает в область $x<0$, то есть открытому граничному условию.

\section{7. Заключение}

В работе изучено влияние электрон-дырочной асимметрии на электронную структуру спиральных краевых состояний в квантовой яме $\mathrm{HgTe} / \mathrm{HgCdTe}$. Получены аналитические выражения для волновых функций и энергетического спектра спиральных состояний, тензора $g$-фактора, матричных элементов оптических переходов между краевыми состояниями с противоположным спином в рамках электро-дипольного механизма. Показано, что при наличии электрон-дырочной асимметрии спектр краевых состояний отклоняется от линейного, и найдены поправки более высоких степеней по волновому вектору. Показано, что электрон-дырочная асимметрия оказывает наибольшее влияние на структуру спиральных состояний для резкой границы с вакуумом, в случае же плавной границы, например, контакта между двумя изоляторами, ее роль существенно снижается. Полученные результаты могут быть использованы при анализе магнито-транспортных явлений и краевого фотогальванического эффекта в квантовых ямах $\mathrm{HgTe} / \mathrm{HgCdTe}$.

\section{Приложение А: Расчет волновых функций краевых состояний при $\mathscr{D}=0$}

В этом разделе мы получим аналитические выражения для волновых функций $\psi_{k_{y} s}$ в случае $\mathscr{D}=0$ и в пределе $\mathscr{D} / \mathscr{B} \ll 1$. Функции $\psi_{k_{y} s}$ будем искать в виде (23), где система уравнений на функции $a_{1,2}$ и $b_{1,2}$ приведена в (29). Будем искать решение системы в виде $a_{1,2}, b_{1,2} \propto e^{-\lambda x}$ с положительными $\lambda$. Подставив решение в систему, найдем корни характеристического уравнения $\lambda_{j}$ и соответствующие им вектора как функции энергии $E$. Можно показать, что граничное условие $\psi_{k_{y} s}(x=0)=0$ может быть удовлетворено только при $E=A k_{y} / \sqrt{1+k_{0}^{2} l_{2}^{2}}$, см. формулу (30).

В пределе $l_{1} \ll l_{2}$ найденной энергии отвечают следующие $\lambda$ :

$$
\lambda_{1}=\lambda_{2}=\frac{1}{l_{1}}, \quad \lambda_{3}=\lambda_{4}^{*}=\frac{1}{l_{2}}-i k_{0} \sqrt{1-\frac{E^{2}}{\delta_{0}^{2}}} .
$$

Из этой формулы видно, что при $|E|<\left|\delta_{0}\right|$ (энергия краевого состояния лежит в объемной щели) меняется только период осцилляций волновой функции, а длина затухания не меняется. При $|E|>\left|\delta_{0}\right|$ начинает увеличиваться длина затухания, и наконец, при $E= \pm \delta_{0} \sqrt{1+\delta_{0}^{2} / \gamma^{2}}$ длина затухания обращается в бесконечность. Как и в случае $\gamma=0$, это происходит в точке касания краевой и объемной дисперсионных ветвей.

Приведем также окончательные выражения для функций $a_{1,2}$ и $b_{1,2}$

$$
\begin{gathered}
a_{1}(x)=v_{1}\left\{e^{-x / l_{1}} \cos \varphi_{1}-e^{-x / l_{2}} \cos \left[k_{0}(E) x-\varphi_{1}\right]\right\}, \\
a_{2}(x)=v_{1} e^{-x / l_{1}} \cos \varphi_{1}-v_{2} e^{-x / l_{2}} \cos \left[k_{0}(E) x-\varphi_{1}-\varphi_{2}\right], \\
b_{1}(x)=e^{-x / l_{1}} \sin \varphi_{1}+v_{1} v_{2} e^{-x / l_{2}} \sin \left[k_{0}(E) x-\varphi_{1}-\varphi_{2}\right], \\
b_{2}(x)=e^{-x / l_{1}} \sin \varphi_{1}+e^{-x / l_{2}} \sin \left[k_{0}(E) x-\varphi_{1}\right] . \quad(\mathrm{A} 2)
\end{gathered}
$$

Здесь

$$
\begin{gathered}
k_{0}(E)=k_{0} \sqrt{1-\frac{E^{2}}{\delta_{0}^{2}}}, \quad \tan 2 \varphi_{1}=\frac{k_{0}(E) l_{2}}{1+k_{y} l_{2}}, \\
v_{1}=\sqrt{\frac{\left|\delta_{0}\right|-E}{\left|\delta_{0}\right|+E}}, \quad v_{2}=\sqrt{\frac{\left|\delta_{0}\right| \sqrt{1+k_{0}^{2} l_{2}^{2}}-E}{\left|\delta_{0}\right| \sqrt{1+k_{0}^{2} l_{2}^{2}}+E}}, \\
\tan \varphi_{2}=\frac{E k_{0} l_{2}}{\sqrt{\delta_{0}^{2}-E^{2}} \sqrt{1+k_{0}^{2} l_{2}^{2}}} .
\end{gathered}
$$

Как было показано в разделе IV В2, в случае $\mathscr{D} / \mathscr{B} \ll 1$ система $(29)$ имеет такой же вид, что и при $\mathscr{D}=0$, только теперь длина $l_{2}$ зависит от энергии по формуле (31). Таким образом, чтобы найти волновые функции краевых состояний в пределе $\mathscr{D} / \mathscr{B} \ll 1$, нужно подставить в формулы (A2) и (A3) длину $l_{2}$, определяемую выражением (31).

\section{Благодарности}

Автор выражает благодарность С.А. Тарасенко за полезные обсуждения. 


\section{Финансирование работы}

Работа выполнена при финансовой поддержке гранта Президента Российской Федерации (проект № МК-2943.2019.2) и гранта Фонда развития теоретической физики и математики „Базис“.

Автор выражает благодарность С.А. Тарасенко за полезные обсуждения.

\section{Конфликт интересов}

Автор заявляет, что у него нет конфликта интересов.

\section{Список литературы}

[1] B.A. Bernevig, T.L. Hughes, S.-C. Zhang. Science 314, 1757 (2006).

[2] M.König, S. Wiedmann, C. Brüne, A. Roth, H. Buhmann, L.W. Molenkamp, X.-L. Qi, S.-C. Zhang. Science 318, 766 (2007).

[3] I. Knez, R.-R. Du, G. Sullivan. Phys. Rev. Lett. 107, 136603 (2011).

[4] A. Roth, C. Brüne, H. Buhmann, L.W. Molenkamp, J. Maciejko, X.-L. Qi, S.-C. Zhang. Science 325, 294 (2009).

[5] G.M. Gusev, Z.D. Kvon, O.A. Shegai, N.N. Mikhailov, S.A. Dvoretsky, J.C. Portal. Phys. Rev. B 84, 121302 (2011).

[6] E.Y. Ma, M.R. Calvo, J. Wang, B. Lian, M. Mühlbauer, C. Brüne, Y.-T. Cui, K. Lai, W. Kundhikanjana, Y. Yang, M. Baenninger, M. König, C. Ames, H. Buhmann, P. Leubner, L.W. Molenkamp, S.-C. Zhang, D. Goldhaber-Gordon, M. A. Kelly, Z.-X. Shen. Nature Commun. 6, 7252 (2015).

[7] S.U. Piatrusha, E.S. Tikhonov, Z.D. Kvon, N.N. Mikhailov, S.A. Dvoretsky, V.S. Khrapai. Phys. Rev. Lett. 123, 056801 (2019).

[8] Y. Tanaka, A. Furusaki, K.A. Matveev. Phys. Rev. Lett. 106, 236402 (2011).

[9] A.M. Lunde, G. Platero. Phys. Rev. B 86, 035112 (2012).

[10] B.L. Altshuler, I.L. Aleiner, V.I. Yudson. Phys. Rev. Lett. 111, 086401 (2013).

[11] J.I. Väyrynen, M. Goldstein, Y. Gefen, L. I. Glazman. Phys. Rev. B 90, 115309 (2014).

[12] M.V. Entin, L.I. Magarill. JETP Lett. 100, 566 (2015).

[13] P.D. Kurilovich, V.D. Kurilovich, I.S. Burmistrov, M. Goldstein. JETP Lett. 106, 593 (2017).

[14] K.E. Nagaev. Phys. Status Solidi (RRL) - Rap. Res. Lett. 12, 1700422 (2018).

[15] K.-M. Dantscher, D.A. Kozlov, M.T. Scherr, S. Gebert, J. Bärenfänger, M.V. Durnev, S.A. Tarasenko, V.V. Bel'kov, N.N. Mikhailov, S.A. Dvoretsky, Z.D. Kvon, J. Ziegler, D. Weiss, S.D. Ganichev. Phys. Rev. B 95, 201103 (2017).

[16] M.V. Durnev, S.A. Tarasenko. Ann. Phys. 1800418 (2019).

[17] Z. Fei, T. Palomaki, S. Wu, W. Zhao, X. Cai, B. Sun, P. Nguyen, J. Finney, X. Xu, D.H. Cobden. Nature Phys. 13, 677 EP (2017).

[18] X.-L. Qi, S.-C. Zhang. Rev. Mod. Phys. 83, 1057 (2011).

[19] M. König, H. Buhmann, L.W. Molenkamp, T. Hughes, C.X. Liu, X.-L. Qi, S.-C. Zhang. J. Phys. Soc. 77, 031007 (2008).

[20] B. Zhou, H.-Z. Lu, R.-L. Chu, S.-Q. Shen, Q. Niu. Phys. Rev. Lett. 101, 246807 (2008).

[21] E.B. Sonin. Phys. Rev. B 82, 113307 (2010).

[22] P.C. Klipstein. Phys. Rev. B 91, 035310 (2015).
[23] V.V. Enaldiev, I.V. Zagorodnev, V.A. Volkov. Pis'ma Zh. Eksp. Teor. Fiz. 101, 94 (2015).

[24] V. Kaladzhyan, P.P. Aseev, S.N. Artemenko. Phys. Rev. B 92, 155424 (2015).

[25] M.V. Entin, M.M. Mahmoodian, L.I. Magarill. EPL (Europhys. Lett.) 118, 57002 (2017).

[26] X. Dai, T.L. Hughes, X.-L. Qi, Z. Fang, S.-C. Zhang. Phys. Rev. B 77, 125319 (2008).

[27] S.A. Tarasenko, M.V. Durnev, M.O. Nestoklon, E.L. Ivchenko, J.-W. Luo, A. Zunger. Phys. Rev. B 91, 081302 (2015).

[28] R. Winkler, L. Wang, Y. Lin, C. Chu. Solid State Commun. 152, 2096 (2012).

[29] M.V. Durnev, S.A. Tarasenko. Phys. Rev. B 93, 075434 (2016).

[30] G.M. Minkov, A.V. Germanenko, O.E. Rut, A.A. Sherstobitov, M.O. Nestoklon, S.A. Dvoretski, N.N. Mikhailov. Phys. Rev. B 93, 155304 (2016).

[31] M.V. Durnev, S.A. Tarasenko. J. Phys.: Condens. Matter 31, 035301 (2019).

[32] M. Entin, L. Magarill. Pis'ma Zh. Eksp. Teor. Fiz. 103, 804 (2016).

[33] X. Marie, T. Amand, P. Le Jeune, M. Paillard, P. Renucci, L.E. Golub, V.D. Dymnikov, E.L. Ivchenko. Phys. Rev. B 60, 5811 (1999).

[34] R. Jackiw, C. Rebbi. Phys. Rev. D 13, 3398 (1976).

[35] B.A. Volkov, O.A. Pankratov. Pis'ma Zh. Eksp. Teor. Fiz. 42, 145 (1985).

Редактор Т.Н. Василевская 